\title{
Innovación sustentable: un camino al desarrollo productivo del Ecuador
}

\section{Sustainable innovation: a path to the productive development of Ecuador}

Dely Nathalia Bravo Donoso

Flavio Roberto Arroyo Morocho

Universidad Central del Ecuador, Ecuador

Autor corresponsal:dnbravo@uce.edu.ec, frarroyo@uce.edu.ec

Fecha de recepción: 20 de Octubre de 2017 - Fecha de aceptación: 15 de Mayo de 2018

Resumen: En los últimos años ha sido evidente el aumento de factores que están perjudicando el planeta, tal es el caso de la contaminación de los suelos, tala de árboles, deshielo de los polos, agujero en la capa de ozono, cambio climático, etc.; estos factores repercuten de manera drástica sobre el globo terráqueo, que ha entrado en un estado de crisis ambiental y social, debido a que las personas no toman conciencia de la importancia que tiene el cuidado al medio ambiente. El mundo vive bajo una sociedad de consumo, en donde lo que prima es el objeto, ya sea para satisfacer una necesidad real o social; en éste contexto la población busca apoderarse de la mayor cantidad de productos, y olvida por completo el impacto ambiental que conlleva la producción, dicho de otro modo el consumidor desconoce por completo la relación entre las variables energía, materiales, residuos y agua que están implícitos en dicho proceso y que repercuten de manera directa sobre el planeta. En este artículo se mencionan aspectos relacionados con la importancia que tiene el implementar un desarrollo sostenible en la transformación social y ambiental para generar una concientización mundial que permita reducir el grado de daño que se está ocasionando al planeta, mediante datos estadísticos que relacionan la diferencia que existiría entre seguir consumiendo los productos tradicionales con respecto a consumir productos que respeten el medio ambiente. Con esto se busca evidenciar que todo el proceso de ciclo de vida del producto debe estar orientado hacia el cuidado del medio ambiente teniendo en cuenta los factores que conllevan a la realización de un producto tales como: producción, análisis de mercado, distribución, diseño, análisis de material, etc.

Palabras Claves: medio ambiente; desarrollo; economía circular; innovación sostenible; sostenibilidad

Abstract: In recent years, there has been an evident the increase of factors that are damaging the planet, such as soil contamination, tree felling, pole melting, the ozone hole, climate change, etc..; These factors have a drastic impact on the world, which has entered in a state of environmental and social crisis, because people are not aware of the importance of care to the environment. The world lives in a consumer society, where what is prime is the object, whether to satisfy a real or social need; In that context, the population seeks to take over the largest quantity of products and completely forgets the environmental impact of production, otherwise the consumer is completely unaware of the relationship between the variables like energy, materials, waste and water, that are implicit in the mentioned process and that have a direct impact on the planet. This article mentions aspects related to the importance of implementing a sustainable development in social and 
environmental transformation to generate a global awareness that allows to reduce the degree of damage that is being caused to the planet, through statistics that relate the difference that There would be a need to continue to consume traditional products to consume products that respect the environment. The aim is to demonstrate that the whole product life cycle process must be geared towards the care of the environment considering the factors that lead to the realization of a product such as production, market analysis, distribution, design, Material analysis, etc.

Key Words: environment; development; circular economy; sustainable innovation; sustainability

\section{Introducción}

Cambiar los paradigmas de la sociedad y crear conciencia en los seres humanos con respecto a la naturaleza y el gran daño que sufre el planeta por el alto grado de industrialización, es un reto que pocos gobiernos han asumido, pues a pesar de los grandes avances tecnológicos y el alto grado de conectividad en el mundo, el desconocimiento en torno al deterioro medioambiental al que se ve expuesto a diario el planeta por la excesiva producción, se mantiene y se sigue creyendo que los recursos naturales son inagotables; lastimosamente, la realidad es distinta, hoy en día es común escuchar que ciertas especies animales se han extinto, y que gran variedad de plantas se encuentran en el mismo peligro debido al cambio climático por el que atraviesa la Tierra, efecto que también influye en la reducción del volumen de agua dulce del que dispone el planeta.

En el Ecuador la situación no es distinta, las cifras de especies en peligro de extinción aumentan, la explotación del Yasuní es un hecho, la tala indiscriminada de árboles se mantiene, y el cambio climático se ha vuelto cada vez más evidente; estas son pruebas suficientes para entender que el país requiere tomar conciencia y generar un cambio, que debe comenzar por los hábitos de consumo que mantiene la sociedad.

Bajo éste contexto, la industria ha buscado medios alternativos para reducir la contaminación durante el proceso de fabricación, el uso y posterior desecho del objeto, y ha dado lugar al producto sustentable que busca contribuir con el planeta a la par de satisfacer las necesidades de los consumidores, es por ello que su proceso de producción incluye diseño, manufactura y comercialización, siempre teniendo en consideración el respeto medioambiental.

El 25 de septiembre de 2015, los líderes mundiales adoptaron un conjunto de objetivos globales para erradicar la pobreza, proteger el planeta y asegurar la prosperidad para todos como parte de una nueva agenda de desarrollo sostenible. La Agenda 2030 para el Desarrollo Sostenible, es un plan de acción en favor de las personas, el planeta y la prosperidad. También tiene por objeto fortalecer la paz universal dentro de un concepto más amplio de la libertad. Reconoce que la erradicación de la pobreza en todas sus formas y dimensiones, incluida la pobreza extrema, es el mayor desafío a que se enfrenta el mundo y constituye un requisito indispensable para el desarrollo sostenible (Naciones Unidas, 2015a).

\section{Metodología}

La ciencia, la tecnología y la innovación son fundamentales para el desarrollo de la capacidad productiva, el aumento de la productividad, la promoción de empresas e industrias competitivas y la recuperación económica (United Nations, 2016). Un país desarrolla 
capacidades para innovar, y la ausencia de tales capacidades resulta en limitaciones para aplicar las tecnologías existentes en todos los sectores, incluidos los de importancia pública, como la salud, la agricultura y el cambio climático (ITU,OHCHR, UNCTAD, UNEP, UNESCO, UNFCCC, UNIDO, WIPO, WMO, 2012).

Uno de los principales desafíos que enfrenta el planeta tiene que ver con el deterioro del medio ambiente y la amenaza del cambio climático. La innovación sustentable es la manera práctica de alcanzar un desarrollo productivo sostenible, razón por la cual resulta necesaria una estrategia de desarrollo que facilite la transición hacia modelos de producción y economías más sustentables.

Con respecto al problema energético que se presenta en el Ecuador es posible buscar para cada proceso productivo una fuente alternativa de energía renovable que no emita gases contaminantes. Teniendo en consideración el sector que más contribuye en la generación de emisiones contaminantes se deberían tomar en cuenta proyectos de movilidad sostenible como es el caso de uso de vehículos eléctricos, la construcción de proyectos de transporte masivo como el metro o el tranvía, y lo más importante diseñar proyecto de transporte pesado que dejen de utilizar energía proveniente de recursos no renovables, todo esto es viable con la implementación de la nueva matriz energética sustentada en la hidroenergía.

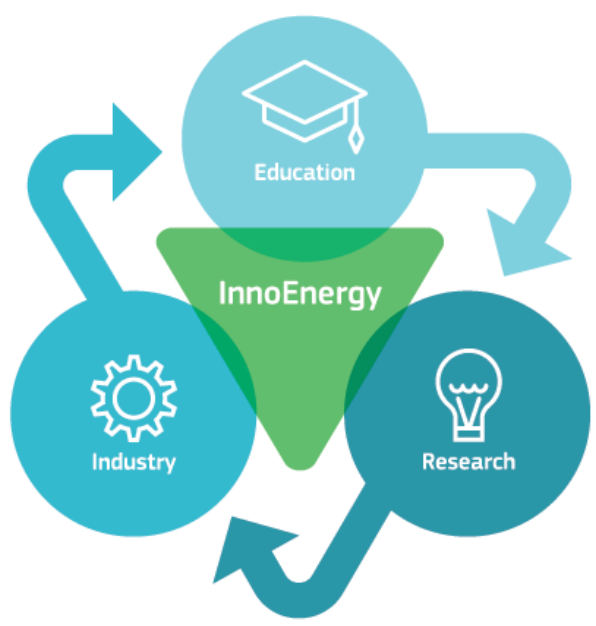

Figura 4. Sustainable energy for all Fuente: (Pavia, 2017)

La transición a una economía baja en carbono es importante para transformar la base industrial ecuatoriana, incluidos los sectores de la energía, la fabricación, el transporte y la construcción. Las soluciones y tecnologías desarrolladas abarcan la optimización de procesos, la implantación de nuevos sistemas, el desarrollo de nuevos productos, el desarrollo de guías de buenas prácticas y centros de conocimiento. La producción de energías renovables y su uso en todos los sectores de la sociedad y nos generará una ventaja competitiva.

La innovación enfocada en la sustentabilidad de los procesos productivos que actualmente contaminan el aire, el agua, el suelo se presenta como la solución más adecuada a los problemas medioambientales que de no solventarse generarán cambios irreversibles en el clima y en el ecosistema del Ecuador. 
Para alcanzar un desarrollo industrial sustentable es necesario que las industrias puedan acceder con facilidad a tecnologías más sustentables. La sociedad poco o nada se preocupa en los medios de fabricación industrial, tampoco prestan atención al proceso de obtención de materia prima, y mucho menos al índice de contaminación que genera el desarrollo y uso de los productos; por tal motivo se debería motivar a la ciudadanía al uso responsable de los objetos, y a un adecuado desecho de los mismos, con miras de reducir los índices de contaminación.

Por otra parte, se conoce que la industria forma parte activa de la economía mundial, especialmente en los países desarrollados, en donde el consumo es elevado pues las necesidades cada día son más exigentes y mayores; es por ello que la producción es altamente contaminante y poco respetuosa con el medioambiente.

El diseño de nuevos productos o servicios debe enfocarse en metodologías como el ecodiseño o la economía circular, es decir, tomar en cuenta todos aspectos ambientales desde la fase del diseño, el proceso de elaboración, y la distribución que deben estar enfocados a cuidar el medio ambiente. Aquí se relaciona otro concepto que es la económica circular, dentro de este círculo todos deben salir ganando, tanto el consumidor como la entidad que genera el producto o servicio, pero principalmente gana el planeta.

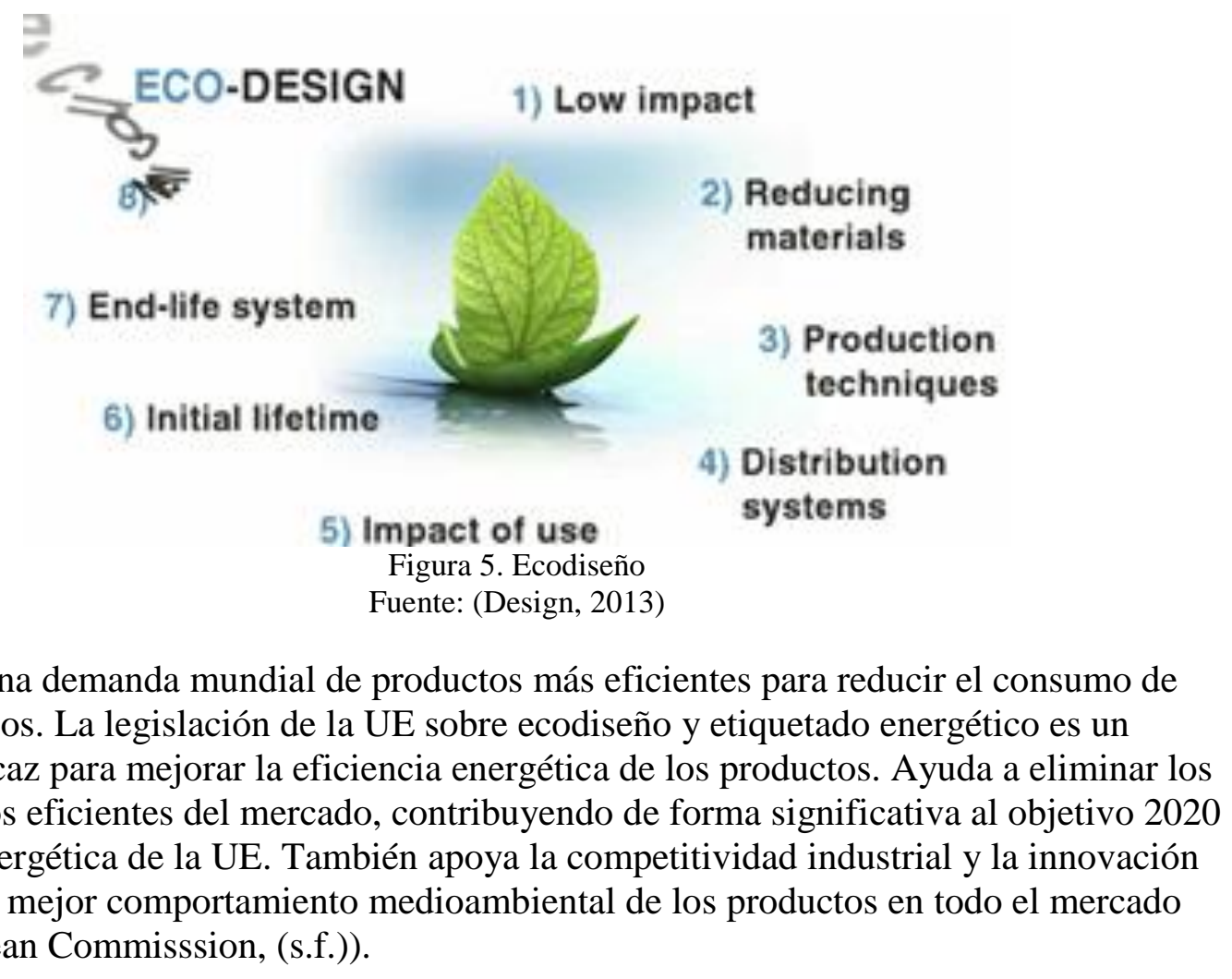

La economía circular representa una gran oportunidad para nuestro país. Se mejorará el uso de los recursos y se aportará un valor agregado a los negocios, englobando, al mismo tiempo, sostenibilidad ambiental, lucha contra el cambio climático y bienestar socioeconómico para las generaciones presentes y futuras. 


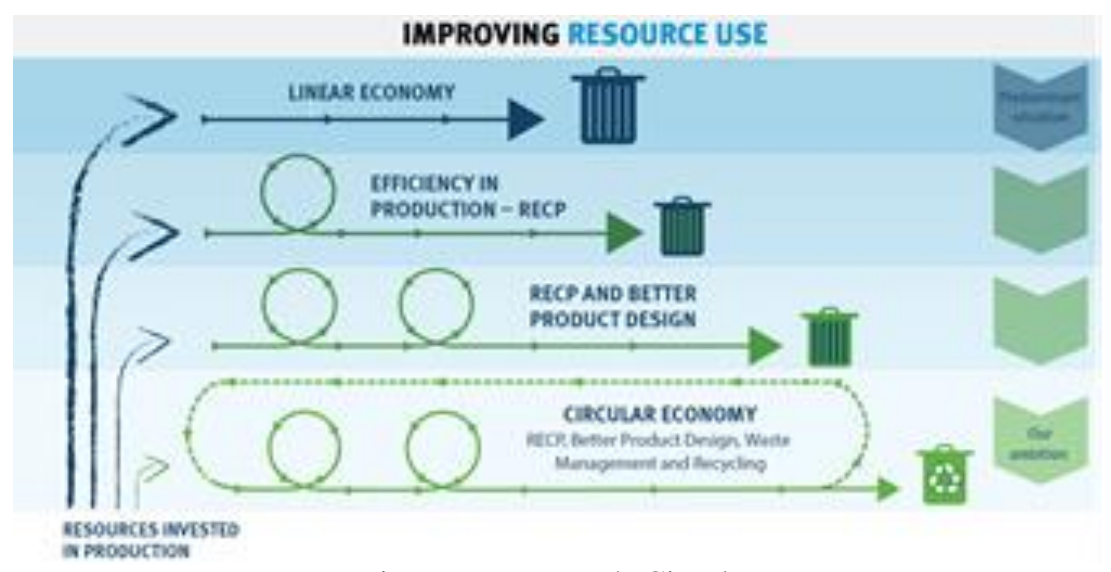

Figura 6. Economía Circular

Fuente: (United Nations Industrial Development Organization, (s.f))

Proyectos de economía circular promueven la sustitución de materiales peligrosos para que el reciclado de los componentes sea más rentable. Las modificaciones propuestas facilitarán las operaciones del mercado de segunda mano y la reparación de aparatos eléctricos y electrónicos (Europea, 2017). Las implementaciones de estos planes de acción han ayudado a evitar más de 3000 toneladas de residuos peligrosos al año en la UE y se ha ahorrado energía y materias primas.

Hoy en día el $71 \%$ de todo el calzado y la ropa Nike incorpora materiales reciclados, y sus diseñadores utilizan 29 materiales de alto rendimiento basados en chatarra de fábrica. Se están implementando y ampliando nuevos procesos de diseño y fabricación, con materiales recuperados a lo largo de la producción y al final de la vida de un producto.

Apple ha adoptado el compromiso de ayudar a reciclar sus productos de forma responsable y para ello ha desarrollado un programa (Apple Renew program) que permite a los consumidores reciclar sus aparatos viejos online o en las tiendas. En el $99 \%$ de los países en los que vende sus productos, Apple desarrolla un Programa de Reutilización y Reciclaje, de manera que cada tienda de distribución en el mundo acepta los productos de Apple para un reciclaje responsable.

Puma dispone de toda una colección de ropa, zapatillas y accesorios certificada por el Crade to Cradle Institute. Los artículos han sido diseñados para ser enteramente reciclados una vez recuperados a través de las tiendas Puma.

MUD Jeans demuestra que todo producto cabe dentro de los principios de la economía circular; los jeans están diseñados para facilitar su reciclaje, pueden comprarse o alquilarse, la empresa los recompra cuando el consumidor decide no usarlos más y los repara y revende como ropa de segunda mano o los recicla para fabricar nuevos jeans.

Lo que se pretende es que Ecuador sea capaz de generar proyectos de mejora e innovación dirigidos hacia un desarrollo sostenible hasta llegar a la implementación de una economía circular. Es un trabajo largo pero el impacto degenerativo que está pasando el planeta amerita medidas extremas y planes de acción inmediatos y que mejor si se parte desde el hogar (Productividad, 2012). 


\section{Técnicas y herramientas}

La innovación sustentable es una respuesta posible a los desafíos que plantea el desarrollo sustentable para las empresas, se entiende como las diferentes acciones que buscan nuevas formas de reducir el impacto medioambiental del desarrollo productivo, de manera que la economía se desarrolle sin comprometer el desarrollo de las futuras generaciones (Rovira \& Hiriart, 2014).

De acuerdo con (SENPLADES, 2015) varios proyectos productivos nacen de la necesidad que se tiene de cuidar el medio ambiente, respetar la naturaleza, vivir en armonía y alcanzar el Sumak Kawsay. Las estrategias de crecimiento deben incorporar todos los tipos de capital, tanto tangibles como intangibles, incluyendo los recursos naturales, el capital humano y físico y la innovación (Rovira \& Hiriart, 2014).

Hoy se mira hacia modelos de desarrollo sostenible que apuesten por una producción con valores, que privilegian al ser humano y al ambiente. Este nuevo proceso de sostenibilidad industrial se fundamenta en varias políticas que reconocen a la naturaleza como sujeto de derechos, con cada política forjada para el desarrollo del Buen Vivir, la economía con valores y la complementariedad pública y privada.

En el camino hacia un modelo de reconversión productiva, se intenta ejecutar una serie de políticas integrales y articuladas para promover la industria verde. Mediante un uso más eficiente de los recursos se puede logar una mejora en la productividad en las empresas, la innovación permite brindar soluciones a problemas ambientales, así como la creación de nuevos mercados.

La innovación sustentable no se limita a la conservación del medioambiente, sino a todos aquellos aspectos que afectan los medios de producción, tales como la mejora de las condiciones de los trabajadores, las mejorar de los sistemas de diseño industrial, la relación con los clientes y la institucionalidad del aparato productivo, entre otros (Rovira \& Hiriart, 2014).

En Ecuador no existen mayor número de proyectos relacionados con innovación sustentable, pues es un tema relativamente nuevo y está en etapa de difusión, sin embargo, existen proyectos pilotos que se están ejecutando. El país se ha centrado principalmente en incentivar en la sociedad el hábito del reciclaje, el mismo que constituiría el primer paso para promover una innovación sustentable tanto para industrias como para la sociedad.

Según (Haro Lenin, 2016) en el caso del país se intenta impulsar acciones para limitar el uso de aparatos ineficientes, como el cambio de lámparas incandescentes por focos ahorradores, programa en el que se invirtieron 20 millones de dólares, lo que significó un ahorro de 101 millones de dólares anuales y la reducción de 226.000 toneladas de $\mathrm{CO}_{2}$.

En el marco del Protocolo de Montreal y con la ayuda de la Organización de las Naciones Unidas para el Desarrollo Industrial (ONUDI), se promueve la reconversión tecnológica y la eliminación de las sustancias agotadoras de la capa de ozono en las industrias; por ejemplo: 
erradicar los CFC y lo mismo con los $\mathrm{HCFC}$ en el sector espumas flexibles, aires acondicionados y automotor (INEC, 2016).

De acuerdo con (RSE, 2015), en el año 2015 se recibieron casi 900 proyectos de innovación sustentable, provenientes de 84 países en diferentes categorías, como moda, alimentación, desarrollo urbano, transporte y salud; entre los ganadores se tiene:

Wecyclers, Nigeria: Este proyecto, consiste en una respuesta a la crisis local de basura. Wecycle tiene una flota de bicicletas que recogen y reciclan la basura de barrios de bajos ingresos, impulsando a las familias a reciclar botellas, latas y plásticos. Por cada kilo que es recolectado, la familia recibe puntos que pueden ser canjeados por minutos de celular, alimentos o utensilios básicos del hogar.

Netafim, Israel: Es un sistema de irrigación de baja tecnología para pequeños agricultores en países en desarrollo, el cual aumenta la cosecha al mismo tiempo que reduce agua y costos, ya que administra agua y nutrientes en cantidades precisas justo en las raíces. Esto minimiza la necesidad de invertir en electricidad e infraestructura.

AirCarbon, Estados Unidos: Una tecnología que convierte los gases de efecto invernadero en material plástico, la cual permite reemplazar a los plásticos basados en petróleo con un producto sustentable que es competitivo en precio y desempeño. Esto se logra en un proceso en el que se captura el carbono del aire. La tecnología ya se ha usado para hacer sillas, bolsas y protectores de teléfonos celulares.

En términos generales, cuando se habla de innovación sostenible la intención es definir aquellos procesos que reúnen múltiples facetas de manera que no ayuden únicamente a la investigación y a la mejora directa sobre el medio ambiente, sino a todos aquellos procesos en los que participan los medios de producción.

El Programa de las Naciones Unidad para el Desarrollo promueve un cambio hacia la sostenibilidad, donde el crecimiento económico beneficie a los más pobres y grupos marginados y evite daños irreversibles al medio ambiente.

\section{Resultados y discusión}

La industria manufacturera es una importante fuente de empleo, en el año 2009 se registraron alrededor de 470 millones de empleos. En 2013, se estima que había más de quinientos millones de empleos en el sector manufacturero (Naciones Unidas, 2015b). El crecimiento económico, el desarrollo social y la acción contra el cambio climático dependen en gran medida de la inversión en infraestructuras, desarrollo industrial sostenible y progreso tecnológico (Naciones Unidas, 2016a).

El consumo y la producción sostenibles consisten en fomentar el uso eficiente de los recursos y la eficiencia energética, infraestructuras sostenibles y facilitar el acceso a los servicios básicos, empleos ecológicos y decentes, y una mejor calidad de vida para todos. Su aplicación ayuda a lograr los planes generales de desarrollo, reducir los futuros costos económicos, 
ambientales y sociales, aumentar la competitividad económica y reducir la pobreza (Naciones Unidas, 2015c). El objetivo del consumo y la producción sostenibles es hacer más y mejores cosas con menos recursos, incrementando las ganancias netas de bienestar de las actividades económicas mediante la reducción de la utilización de los recursos, la degradación y la contaminación durante todo el ciclo de vida, logrando al mismo tiempo una mejor calidad de vida (Naciones Unidas, 2015c).

Hay muchos aspectos del consumo que, con sencillos cambios, pueden tener un gran impacto en el conjunto de la sociedad. Por ejemplo, cada año, alrededor de un tercio de todos los alimentos producidos - el equivalente a 1.300 millones de toneladas, por un valor aproximado de 1 billón de dólares - termina pudriéndose en los cubos de basura de los consumidores y los minoristas, o deteriorándose a causa de las deficientes prácticas de recolección y transporte, algo que las empresas deben solucionar (Naciones Unidas, 2016b).

En lo que respecta a los consumidores, los hogares consumen el $29 \%$ de la energía mundial y contribuyen al $21 \%$ de las emisiones de $\mathrm{CO}_{2}$ resultantes. Sin embargo, si toda la población mundial pasara a utilizar bombillas de alto rendimiento energético, ahorraríamos 120.000 millones de dólares al año. La contaminación de las aguas es también una cuestión apremiante que exige una solución sostenible. Estamos contaminando el agua más rápidamente de lo que la naturaleza puede reciclar y purificar en los ríos y los lagos (Naciones Unidas, 2016b).

Ante la rápida evolución del panorama económico mundial y el aumento de las desigualdades, el crecimiento sostenido debe incluir una industrialización que en primer lugar, ofrezca oportunidades a todas las personas, y en segundo lugar, cuente con el respaldo de la innovación y de infraestructuras resilientes. El nacimiento de nuevas industrias supone para muchos de nosotros una mejora del nivel de vida. Además, si las industrias aspiran a la sostenibilidad, este enfoque impactará positivamente en el medio ambiente. El cambio climático nos afecta a todos (Naciones Unidas, 2016a).

La energía es el principal contribuyente al cambio climático, y representa alrededor del $60 \%$ del total de emisiones de gases de efecto invernadero a nivel mundial (Naciones Unidas, 2015d). Durante muchos decenios, los combustibles fósiles como el carbón, el petróleo o el gas han sido las principales fuentes de producción de electricidad, pero la quema de combustibles con alto contenido en carbono produce grandes cantidades de gases de efecto invernadero, que contribuyen al cambio climático y tienen efectos perjudiciales para el bienestar de la población y el medio ambiente (Naciones Unidas, 2016d).

En la última década el Ecuador, se ha visto expuesto al incremento de un $39 \%$ en el consumo de productos elaborados importados; este fenómeno es el resultado del paradigma social que envuelve a los ecuatorianos, quienes consideran que lo extranjero tiene mejor calidad y precio accesible; lo que genera que el producto nacional no pueda competir y sea poco atractivo al mercado nacional.

Según el informe del Banco Central del Ecuador, la balanza comercial ha dejado un déficit de aproximadamente 184 millones de dólares en los últimos años, por ello se ha buscado 
generar conciencia en el consumidor a través del slogan "Primero lo Nuestro", que trata de incrementar la demanda de productos nacionales en el mercado ecuatoriano. A criterio de Guillermo Ibarra, gerente propietario del Comisariato Guivas, el ecuatoriano consume producto nacional siempre y cuando su precio sea accesible, más no porque se sienta identificado con una marca nacional.

La globalización obliga a las empresas a nivel regional a enfrentar importantes retos para mejorar sus indicadores de productividad. La industria ecuatoriana no debe quedar rezagada de esta transformación que permitirá en un futuro cercano incrementar su crecimiento económico. Si bien los modelos de producción existentes pueden generar un notable crecimiento económico, no se debe dejar de lado las graves consecuencias que se han marcado en el medioambiente, ocasionando consecuencias negativas en la calidad de vida de los habitantes de las diferentes regiones del planeta.

La creación de nuevos mercados y modelos de producción juegan un rol fundamental en el desafío de conciliar el crecimiento económico y el cuidado del medioambiente. Según datos de estadísticas ambientales, el $80 \%$ de las empresas ecuatorianas no invierten en el desarrollo de programas de protección ambiental ni cuentan con estudios que busquen reducir el impacto que genera la producción y el producto. En base a una encuesta realizada (Villacís, 2015), manifiesta que el $98 \%$ de las empresas no tienen sistemas de gestión ambiental ni cuentan con certificaciones medioambientales en sus organizaciones.

De las industrias existentes en el país, se conoce que el sector minero invierte el $61,4 \%$ en equipos e instalaciones para el tratamiento de aguas residuales; el 33,7\% de las empresas invierte en equipos que reducen las emisiones contaminantes, el 19,5\% busca medios para reducir el consumo de energía y el 18,5\% en el ahorro de agua. En menor porcentaje, se tiene que el 9,3\% busca reducir la generación de desechos y apenas el 1,4\% busca reducir ruidos y vibraciones (INEC, 2015a).

Del porcentaje de industrias involucradas en aspectos medioambientales, la mayoría solo se limita a proyectos de reciclaje o concientización social, algo que no está mal, sin embargo, para generar un cambio de paradigma en la sociedad, no basta con el consumo de productos verdes, ya que, a pesar de generar menor impacto medioambiental, siguen siendo contaminantes. Es por ello, que se debe reconsiderar la importancia que tiene una adecuada gestión del diseño en la producción, y tomar el ejemplo de países europeos que han podido implementar un diseño de innovación ambiental en todo aspecto, pues es evidente que al existir nuevas necesidades el consumo es mayor.

Las industrias en su afán de competir y posicionarse en el mercado ofrecen más y mejores productos para satisfacer las necesidades de sus clientes, pero descuidan el tema ambiental, pues muchas veces lo consideran un factor de impedimento o simplemente un gasto extra dentro de la producción.

Según la Organización Mundial para la Salud (OMS) después de haber reunido datos de 3 000 ciudades en el año 2015, las regiones que más sufren de contaminación por emisión de $\mathrm{CO}_{2}$ son las ciudades de países en vías de desarrollo. 
En el año 2015 el Ecuador registró 46 millones de toneladas de $\mathrm{CO}_{2} \mathrm{EP}$ (energía primaria), en la Figura 1, se puede observar que el mayor porcentaje de emisiones en el país proviene del sector de Transporte con un 39\% del total del valor, seguido por las Centrales Eléctricas con un $14 \%$ y las Industrias con un $13 \%$.

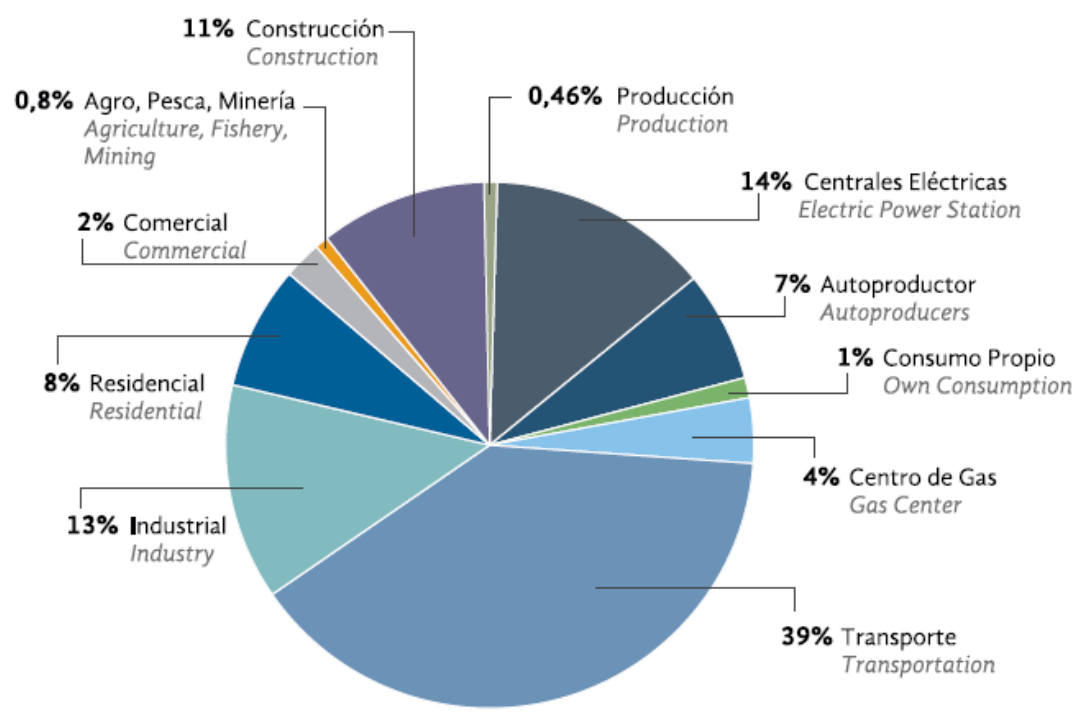

Figura 1. Emisiones de $\mathrm{CO}_{2}$ por Sector

Fuente: (Ministerio Coordinador de Sectores Estratégicos, 2015)

Dejar de utilizar energía proveniente de fuentes no renovables es complicado; ya que, el país depende casi en su totalidad del petróleo y sus derivados, es la mayor fuente de ingresos económicos, sin embargo, es uno de los contaminantes potenciales que tiene Ecuador (Becerra, 2014).

De estudios realizados sobre la generación de emisiones en el Distrito Metropolitano de Quito, el sector con el mayor aporte a las emisiones es el de transporte, con 2.469,0 $\mathrm{Gg} \mathrm{CO}_{2}$, lo cual corresponde al 50.4\% del total. Le siguen en importancia las Industrias de la Energía, subsector Residencial e Industrias Manufactureras y de la Construcción. En la tabla 1 se presenta el detalle del consumo de combustibles y las emisiones de $\mathrm{CO}_{2}$ para cada uno de los sectores mencionados. El 50.4\% de emisiones de $\mathrm{CO}_{2}$ proviene del transporte terrestre debido al mayor número de vehículos que circulan por la cuidad.

Tabla 1. Consumo de combustibles (TJ) y Emisiones $\mathrm{CO}_{2}$ en el DMQ, 2011.

\begin{tabular}{|c|c|c|c|c|c|}
\hline \multirow[t]{2}{*}{ Fuentes } & \multirow[t]{2}{*}{ Detalle fuentes } & \multicolumn{2}{|c|}{$\begin{array}{c}\text { Consumo de } \\
\text { combustibles }\end{array}$} & \multicolumn{2}{|c|}{$\begin{array}{c}\text { Emisión } \\
\mathrm{CO}_{2}\end{array}$} \\
\hline & & (TJ) & $\%$ & Gg & $\%$ \\
\hline Industrias de la Energía & $\begin{array}{l}\text { Energía eléctrica consumida en el } \\
\text { DMQ. }\end{array}$ & 9933 & $17 \%$ & 1564 & $31,9 \%$ \\
\hline $\begin{array}{l}\text { Industrias Manufact. y de } \\
\text { la Construcc. }\end{array}$ & $\begin{array}{l}\text { Consumo combustibles industrias y } \\
\text { comercios. }\end{array}$ & 4080 & $7 \%$ & 2789 & $5,7 \%$ \\
\hline Transporte Terrestre & $\begin{array}{l}\text { Consumo de combustibles de } \\
\text { vehículos públicos y privados. }\end{array}$ & 35140 & $60 \%$ & 2469 & $50,4 \%$ \\
\hline Residencial & $\begin{array}{l}\text { Consumo de GLP doméstico en el } \\
\text { DMQ. }\end{array}$ & 9405 & $16 \%$ & 590,2 & $12 \%$ \\
\hline & Total & 58559 & 100 & 4902 & $100 \%$ \\
\hline
\end{tabular}


Más de la mitad de países del mundo presentan problemas ambientales graves que conlleva al aumento de enfermedades pulmonares. Pekín capital de China es una de las ciudades con mayor grado de emisión de $\mathrm{CO}_{2}$, las personas que habitan ahí tienen que salir de sus casas con mascarillas porque es tan grande el nivel contaminación que parece que la cuidad está dentro de una nube o niebla. Como se puede observar en la Figura 2, de los valores históricos obtenidos del banco mundial sobre emisiones de $\mathrm{CO}_{2}$ en el planeta nos damos cuenta que los valores siguen incrementándose razón por la cual el cambio debe darse de inmediato para precautelar éstas y las futuras generaciones.

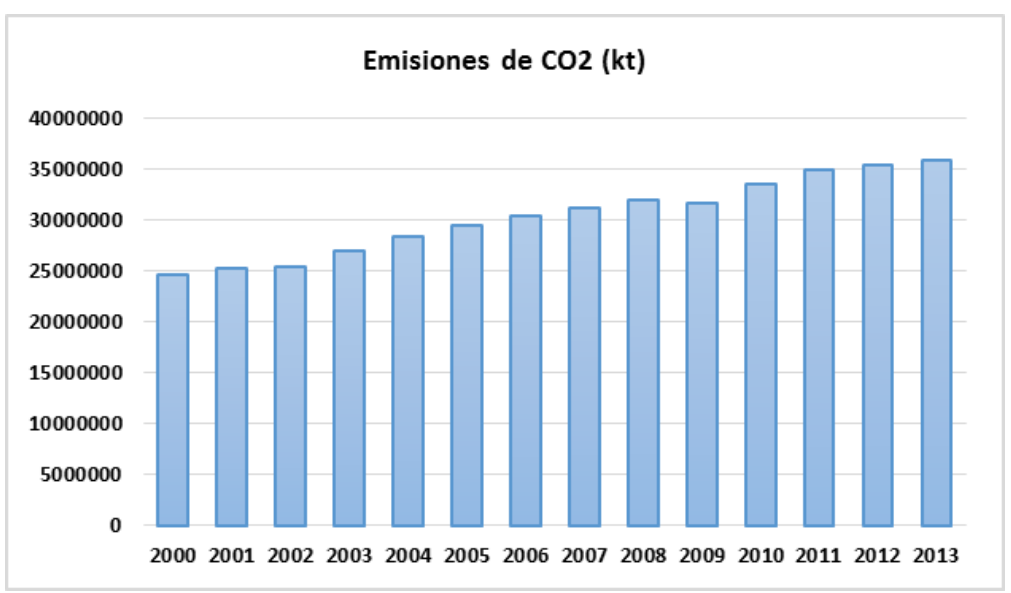

Figura 2. Histórico emisiones de $\mathrm{CO}_{2}$ en el mundo Fuente: (Banco Mundial, 2017)

En el año 2013 el planeta presentó 35.848 .592 kt de $\mathrm{CO}_{2}$ y para el año 2030 se proyectan que serán 51.301 .604 kt, es decir, crecerá en un 43\%, estos son datos que revelan lo importante que es tomar medidas al respecto, pues es una realidad difícil de evadir.

Ecuador y sus niveles de emisión de $\mathrm{CO}_{2}$ en comparación con los niveles mundiales aportan con el $0,025 \%$ lo que puede parecer mínimo, pero a nivel nacional ese porcentaje representa miles de enfermedades, reducción de la calidad del aire, entre otros. En la Figura 3 se puede observar el incremento anual de emisiones de $\mathrm{CO}_{2}$ en Ecuador.

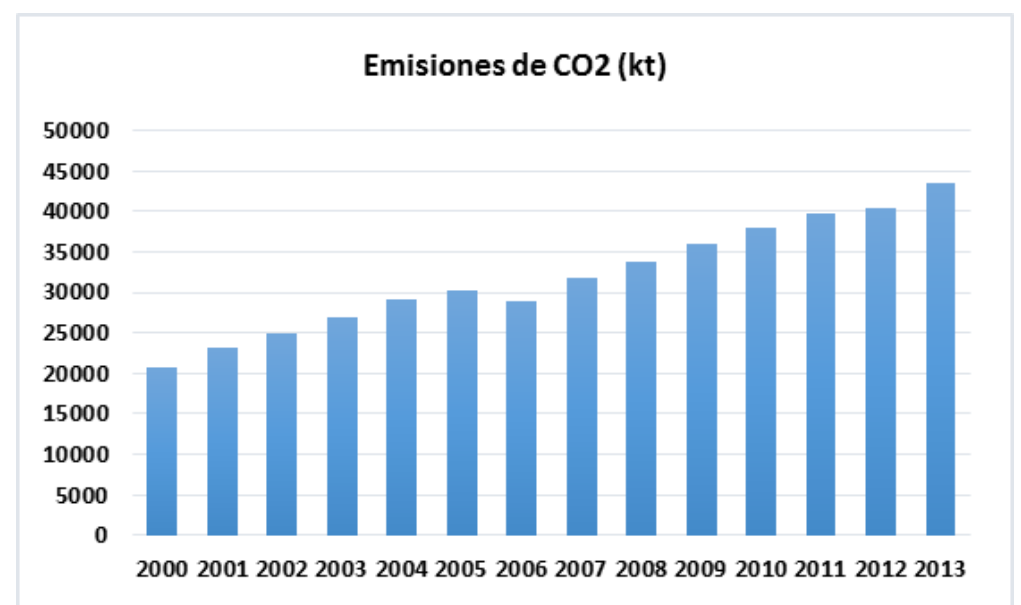


Figura 3. Emisión de $\mathrm{CO}_{2}$ del Ecuador

Fuente: (Banco Mundial, 2017)

Partiendo de los estudios realizados la segunda fuente de emisión de $\mathrm{CO}_{2}$ corresponde a las industrias. Se conoce que existe sinnúmero de industrias en el Ecuador cuyos porcentajes de contaminación son elevados, entre ellas se tiene:

La industria azucarera: presenta $76 \%$ de efectos contaminantes sobre el agua derivados de su elevado consumo energético, sus descargas de alta temperatura y gran contenido de materia orgánica (bagazo, cachaza y vinazas). Además, contribuye al 15\% de la contaminación del aire por la utilización de combustóleo y bagazo, careciendo totalmente de equipos de control de emisiones.

La industria minero-cuprífera: presenta el $90 \%$ de efectos contaminantes del agua por descargas ácidas, de metales, cianuros de sodio, materiales reactivos, aceites lubricantes usados y sólidos suspendidos, y $18 \%$ del aire por partículas de polvo derivadas de sus procesos.

La industria siderúrgica: representa al $45 \%$ a la contaminación al agua con descargas ácidas y amoniacales; al aire con polvos, gases y humos provenientes del carbón y gas natural en procesos de combustión ineficientes (INEC, 2016).

La industria del cuero: genera el 36\% de residuos de "descarne", "raspa", polvo de piel cromada y recorte; además, contamina el agua con sales, cromo, materia orgánica, grasas, taninos vegetales y sintéticos, y el aire con polvos, gases y humos.

La industria textil: es hoy el segundo mayor contaminador de agua potable después de la agricultura, y muchas empresas de moda explotan a los trabajadores textiles en los países en desarrollo.

La minería: los riesgos derivan de la fase de explotación, principalmente de la operación de presas de jales. La misma puede generar escurrimientos y arrastres de residuos minerometalúrgicos peligrosos de alta afectación ambiental, así como la descarga de aguas residuales en cuerpos receptores. Igual ocurre en los procesos de beneficio de minerales, que pueden tener efectos ambientales negativos a través de sus aguas residuales, materiales y sustancias peligrosas y, en algunos casos, emisiones a la atmósfera (Villacís, 2015).

La actividad petrolera: involucra el $84 \%$ de acciones de grandes dimensiones que afectan drásticamente al ambiente. Ello es particularmente cierto con relación a las actividades de refinación y petroquímicas que, aunque se convierten en un importante estímulo a la formación de polos industriales, muestran por lo general niveles altos de contaminación, así como de deterioro de su entorno natural.

La generación de residuos, a nivel mundial, constituye uno de los mayores problemas ambientales de nuestro siglo. Según el Censo de Población y Vivienda del año 2010, la población del Ecuador era de 14.483.499 millones de habitantes, registrándose que un $77 \%$ de los hogares elimina la basura a través de carros recolectores y el restante $23 \%$ la elimina de 
diversas formas, así por ejemplo la arroja a terrenos baldíos o quebradas, la quema, la entierra, la deposita en ríos acequias o canales, etc. (Ministerio del Ambiente, 2010).

Según datos provistos por el Programa Nacional de Gestión integral de Desechos Sólidos, el MIDUVI y otras instituciones, se determinó que el servicio de recolección de residuos sólidos tiene una cobertura nacional promedio del $84.2 \%$ en las áreas urbanas y de $54.1 \%$ en el área rural, la fracción no recolectada contribuye directamente a la creación de micro basurales descontrolados (Ministerio del Ambiente, 2010).

En el Ecuador se recolectaron, durante del 2014, 11.203,24 toneladas diarias de residuos sólidos. Siendo la Costa, la región que mayor recolección registró con 6.229,92 toneladas diarias. En el 2015, a nivel nacional el 39,40 \% de los hogares ecuatorianos clasificó los residuos, 14,24 puntos porcentuales más que lo registrado en el 2010 (INEC, 2015b).

\section{Conclusiones}

En el trabajo se han detallado una serie de oportunidades concretas aún no han sido desarrolladas pero con un gran potencial a futuro como es el caso del uso de energías renovables y tecnologías modernas en el sector de la industria, la aplicación de nuevos modelos de transporte sustentable permitirán reducir significativamente las emisiones de $\mathrm{CO}_{2}$ que como se indicó tienen un crecimiento acelerado.

La actividad productiva es uno de los pilares fundamentales en el desarrollo económico de un país, sin embargo, el excesivo consumo de recursos naturales y la gran generación de residuos son los principales factores que ocasionan al deterioro del medioambiente provocando que el desarrollo económico no tenga una visión sustentable.

Para contribuir con el cuidado medioambiental se debe reducir los desechos que generamos, esto puede lograrse de muchas maneras, desde asegurarnos de no tirar alimentos hasta reducir el consumo de plástico, que es uno de los principales contaminantes del océano. Llevar una bolsa reutilizable y reciclar las botellas de plástico son algunas de las formas de contribuir cada día.

Se debe proceder de forma reflexiva a la hora de comprar y optar por una opción sostenible siempre que sea posible. Si realizamos nuestras compras a proveedores locales y sostenibles, podemos marcar la diferencia y ejercer presión para que las empresas adopten prácticas sostenibles.

Implementar proyectos de energías renovables en Ecuador es un gran paso para reducir los niveles de contaminación, pero estos proyectos deben ir de la mano con la innovación sustentable que permitirá generar proyectos industriales que se preocupen y maximicen la eficiencia en el uso de recursos naturales desde la fase del diseño hasta su disposición final. La energía hidráulica debe ser la principal fuente de generación eléctrica para utilización de los diferentes sectores económicos de país, con lo que se reducirán los niveles de contaminación actual. 
Replicar planes de acción sostenible desarrollados y ejecutados por países o empresas de un mayor desarrollo económico y tecnológico debe ser el objetivo principal de las autoridades en el Ecuador. Al disponer información clara de los proyectos que se han desarrollo permitirá a la industria ecuatoriana involucrase en un desarrollo más sustentable que permita cuidar el medioambiente y posteriormente ser gestores de proyectos de innovación sustentable.

La innovación sustentable es una herramienta adecuada para diseñar o mejorar productos o servicios más amigables con el medioambiente. La economía circular extiende la vida de los productos mediante la reparación, reutilización y re manufacturación de los mismos o manufacturación de nuevos productos que utilizarán de materia prima residuos del producto en desuso.

Realizar cambios en los sistemas productivos del país enfocado en la eficiencia de materiales y energía contribuye a crear un modelo de sociedad más sostenible que beneficie a una mejor calidad de vida. El cambio hacia un modelo económico más sustentable supondría una mejora en la productividad para las industrias en el Ecuador.

Una economía circular reduce la dependencia de los recursos y el uso de los recursos, incluida la energía, reduciendo así los costos de producción, reduciendo la exposición al mercado y limitando los costos derivados de la extracción y generación de recursos. Además, conduce a la introducción de métodos económicamente viables para reducir la contaminación y separar los residuos dañinos de los residuos reutilizables

De residuo a recurso, se debe tomar en cuenta que los residuos pueden ser una fuente inagotable de recursos e ingresos, como se detalló existen empresas que están apostando por el uso de residuos como materia prima de nuevos productos con lo que se logra reducir la generación de residuos y el uso de recursos que afectan el medioambiente.

\section{Bibliografía}

Banco Mundial. (1 de Mayo de 2017). Datos. Obtenido de http://datos.bancomundial.org/indicador/EN.ATM.CO2E.KT

Becerra, R. A. (Junio de 2014). Propuesta de un Plan Nacional que incentive y regule la eficiencia energética. Obtenido de http://bibdigital.epn.edu.ec/bitstream/15000/7490/1/CD-5609.pdf

Design, S. S. (Dirección). (2013). Design thinking - Tutorial on Eco-Design [Película].

Europe economics. (2015). the economic review of the industrial design in Europe. London: Europe Economics.

European Commisssion. ((s.f.)). Crecimiento. Mercado interior, industria, emprendimiento y Pymes. Obtenido de Ecodesign: http://ec.europa.eu/growth/industry/sustainability/ecodesign_es

Haro Lenin, O. J. (2016). Factor Anual de Emisión de CO2 Producido por el Parque Generador 
del Sistema Nacional Interconectado del Ecuador, Mediante la Aplicación de la Metodología de la Convención Marco Sobre el Cambio Climático UNFCCC, para el Periodo 2009-2014. Revista Politécnica, 7.

INEC. (2015a). Directorio de empresas. Obtenido de http://www.ecuadorencifras.gob.ec/directoriodeempresas/

INEC. (2015b). Información Ambiental en Hogares. Obtenido de INEC: http://www.ecuadorencifras.gob.ec//documentos/webinec/Encuestas_Ambientales/Hogares/Hogares_2015/Presentacion\%20enemdu\%202015 $\% 2025 \% 2001 \% 202016 . p d f$

INEC. (Septiembre de 2016). Sistema de Indicadores de la Producción. Obtenido de Instituto Nacional de Estadisticas y Censos: http://www.ecuadorencifras.gob.ec/documentos/webinec/Estadisticas_Economicas/IPI-M/Septiembre-

2016/Presentacion_Resultados_IPI_M_Septiembre_2016.pdf

ITU, OHCHR, UNCTAD, UNEP, UNESCO, UNFCCC, UNIDO, WIPO, WMO. (2012). Science, technology and innovation for sustainable development in the global partnership for development beyond 2015. Geneva: UN System Task Team on the Post-2015 UN Development Agenda.

Ministerio Coordinador de Sectores Estratégicos. (2015). Balance energético nacional 2014. Quito: Ministerio Coordinador de Sectores Estratégicos.

Ministerio del Ambiente. (2010). Programa Nacional para la Gestión Integral de Desechos Sólidos - PNGIDS ECUADOR. Obtenido de http://www.ambiente.gob.ec/programa-pngidsecuador/

Naciones Unidas. (2015a). Proyecto de documento final de la cumbre de las Naciones Unidas para la aprobación de la agenda para el desarrollo después de 2015. Ginebra: Naciones Unidas.

Naciones Unidas. (25 de Septiembre de 2015b). Objetivos de desarrollo sostenible. Obtenido de Objetivo 9: Construir infraestructuras resilientes, promover la industrialización inclusiva y sostenible $\quad \mathrm{y}$ fomentar la innovación: http://www.un.org/sustainabledevelopment/es/infrastructure/

Naciones Unidas. (25 de Septiembre de 2015c). Objetivos de desarrollo sostenible. Obtenido de Objetivo 12: Garantizar modalidades de consumo y producción sostenibles: http://www.un.org/sustainabledevelopment/es/sustainable-consumption-production/

Naciones Unidas. (25 de Septiembre de 2015d). Objetivos de desarrollo sostenible. Obtenido de Objetivo 7: Garantizar el acceso a una energía asequible, segura, sostenible y moderna para todos: http://www.un.org/sustainabledevelopment/es/energy/

Naciones Unidas. (2016a). Industria, innovación e infraestructura: por qué es importante. Ginebra: 
Naciones Unidas.

Naciones Unidas. (2016b). Producción y consumo responsables: por qué son importantes. Ginebra: Naciones Unidas.

Naciones Unidas. (2016d). Energía asequible y no contaminante: por qué es importante. Ginebra: Naciones Unidas.

Pavia, D. (2017). InnoEnergy. SETIS magazine, 23-26.

Productividad, M. d. (Noviembre de 2012). Pais Productivo. Obtenido de http://www.industrias.gob.ec/wpcontent/uploads/downloads/2012/11/pais_productivo2.pdf

Rovira, S., \& Hiriart, C. (2014). Innovación sustentable: espacios para mejorar la competitividad de las pymes argentinas. Santiago de Chile: CEPAL.

RSE, C. d. (2015). Expok. Obtenido de http://www.expoknews.com/10-increibles-innovacionessustentables/

SENPLADES. (2015). Plan Nacional para el Buen Vivir. Obtenido de http://www.planificacion.gob.ec/wpcontent/uploads/downloads/2012/07/Plan_Nacional_para_el_Buen_Vivir_(version_resu mida_en_espanol).pdf

United Nations. (2016). Investment, innovation and entrepreneurship for productive capacity building and sustainable development. Geneva: United Nations Conference on Trade and Development.

United Nations Foundation. (s.f.). What we do: Sustainable Energy for All. Obtenido de (s.f): http://www.unfoundation.org/what-we-do/campaigns-and-initiatives/sustainable-energyfor-all/

United Nations Industrial Development Organization. ((s.f)). Circular economy. Obtenido de http://www.unido.org/what-we-do/cross-cutting-services/circular-economy.html

Villacís, B. (24 de Agosto de 2015). Instituto Nacional de Estadísticas y Censos. Obtenido de El $80 \%$ de las empresas del Ecuador no invierten en protección ambiental: http://www.ecuadorencifras.gob.ec/el-80-de-las-empresas-en-ecuador-no-invierten-enproteccion-ambiental/ 